\title{
Glicocorticóides e síndrome metabólica: aspectos favoráveis do exercício físico nesta patofisiologia
}

\author{
J. Rodrigo Pauli \\ Luciana Souza \\ Gustavo Rogatto \\ Ricardo Gomes \\ Eliete Luciano
}

https://doi.org/10.5628/rpcd.06.02.217

\author{
Universidade Estadual de Campinas \\ Faculdade de Ciências Médicas \\ Laboratório de Sinalização Celular \\ Campinas, Brasil
}

\section{RESUMO}

A síndrome metabólica tem diversas similaridades com a síndrome de Cushing (intolerância à glicose, resistência à insulina, hipertensão, dislipidemia, obesidade central), sugerindo que anormalidades no metabolismo dos glicocorticóides estão associadas com a síndrome metabólica. Por outro lado, a prática regular de atividade física tem sido recomendada para a prevenção e a reabilitação de doenças cardiovasculares e outras doenças crônico-degenerativas. Estudos epidemiológicos têm demonstrado relação direta entre inatividade física e presença de múltiplos fatores de risco como os encontrados na síndrome metabólica. Os efeitos benéficos do exercício físico têm sido demonstrados na prevenção e no tratamento da hipertensão arterial, na resistência à insulina, no diabetes, na dislipidemia e na obesidade. Nesse contexto, esta revisão tem o intuito de discutir os efeitos metabólicos dos glicocorticóides e a associação da ação destes esteróides com as características da síndrome metabólica. Além disso, vamos explorar os mecanismos pelos quais a atividade física pode favorecer o metabolismo dos usuários de glicocorticóides.

Palavras-chave: glicocorticóides, síndrome metabólica, exercício físico.

\begin{abstract}
Metabolic syndrome and glucocorticoid: Favorable aspects of physical exercise on this pathophysiology.

The metabolic syndrome has several similarities with Cushing's syndrome (impaired glucose tolerance, insulin resistance, hypertension, dyslipidemia, central obesity) suggesting that abnormalities in the glucocorticoid metabolism have a link with the metabolic syndrome. On the other hand, regular physical activity provides several health benefits helping in prevention and rehabilitation of cardiovascular and other chronic diseases. Epidemiological studies have been demonstrating a direct relationship between physical inactivity and the multiple risk factors such as those found in the metabolic syndrome. Otherwise, it has been demonstrated the physical exercise benefit to prevent and treat arterial hypertension, insulin resistance, diabetes, dislipidemy, and obesity. This review will discuss the metabolic effects in the context of glucocorticoid metabolism and establish the association of glucocorticoid action with the features of the metabolic syndrome, specially obesity and insulin resistance. Moreover, we will explore how physical activity promotes favorable physiologic adaptations improving quality of life in the abnormalities metabolic association with the excess of glucocorticoid.
\end{abstract}

Keys Words: glucocorticoid, metabolic syndrome and physical exercise. 


\section{INTRODUÇÃO}

O uso de glicocorticóides (GCs) ocorre com freqüência na população, sendo uma das drogas mais prescritas e utilizadas em todo mundo, em várias condições clínicas (1). Constituem indicação absoluta e permanente nos pacientes com insuficiência adrenal, quando utilizam doses de reposição para restabelecer a homeostase do organismo. Na maioria dos casos, eles são usados em crise de asma brônquica, como antiflamatórios, sendo necessárias doses suprafisiológicas, o que promove o aparecimento de muitos efeitos adversos como, por exemplo, atrofia muscular, osteoporose, supressão do sistema imunológico e funcional do eixo hipotálamo-hipófise-adrenais $(2,3,4)$.

O excesso de GCs tem sido também associado com a síndrome metabólica. Na síndrome de Cushing, o aumento na secreção de GCs devido a um adenoma pituitário conduz a obesidade central, hipertensão, hiperlipidemia e intolerância à glicose, um grupo de anormalidades relacionadas com a síndrome metabólica (5). Além disso, a administração clínica destes esteróides no tratamento crônico e agudo de doenças inflamatórias tem sido associada com efeitos metabólicos adversos, tais como pressão sistólica elevada, níveis aumentados de lipídeos circulantes e resistência à insulina, como observado na síndrome metabólica (6).

$\mathrm{Na}$ tentativa de minimizar estes efeitos colaterais realizam-se terapias locais, intervalos na utilização da droga, suplementação com cálcio, vitamina D3, administram-se hormônio do crescimento e estrogênio em humanos (3) e, recentemente, tem sido usado oligonucleotídeo antisense para o receptor de glicocorticóide (GR) em animais (7), entre outras. Por outro lado, estudos com modelos de treinamento físico, no sentido de prevenção da resistência à insulina, bem como das desordens metabólicas, ou ainda, da redução dos possíveis efeitos catabólicos resultantes da administração de glicocorticóide são raros na literatura. Nesse sentido, o objetivo do presente estudo foi realizar uma revisão bibliográfica para investigar a ação dos glicocorticóides sobre o metabolismo e a sua relação com a síndrome metabólica e, ainda, verificar a maneira pela qual o exercício físico pode atuar na prevenção e tratamento das anormalidades relacionadas ao uso de glicocorticóide.

\section{ASSOCIAÇÃO CLÍNICA ENTRE GLICOCORTICÓIDES E SÍNDROME METABÓLICA}

O conceito de síndrome metabólica tem existido há pelo menos 80 anos (8). No entanto, nas últimas duas décadas, o aumento do número de pessoas com síndrome metabólica, distribuídas pelo mundo todo, tem ocupado espaço de destaque no cenário científico. Este aumento está associado com a epidemia global de obesidade e diabetes, e com elevado risco de doenças cardiovasculares associadas à síndrome metabólica (9).

A síndrome metabólica é também conhecida como síndrome $\mathrm{X}$, síndrome da resistência à insulina, quarteto letal e síndrome plurimetabólica (10). Apesar das diferentes definições e critérios para classificá-la, entre os distúrbios metabólicos comumente presentes estão a intolerância à glicose, resistência à insulina, obesidade central, dislipidemia e hipertensão, todos bem documentados fatores de risco para doenças coronarianas (10).

Evidências clínicas têm demonstrado associação entre o metabolismo anormal de GC e a síndrome metabólica. Os níveis de cortisol plasmáticos aumentados, encontrados com o envelhecimento, estão relacionados com uma ou mais características da síndrome metabólica (11). Verificou-se que, tanto a razão da secreção, como as liberações periféricas de cortisol nesses pacientes foram positivamente correlacionadas com a pressão sanguínea sistólica, glicose e insulina de jejum. Outros estudos também reportaram correlação entre o aumento da atividade dos GCs e a redução da sensitividade periférica à insulina, níveis elevados de glicose plasmática e hipertensão $(12,13,14)$.

O padrão de deposição de gordura central da síndrome metabólica assemelha-se muito ao que ocorre na síndrome de Cushing, ou em indivíduos que foram administrados com glicocorticóide sintéticos, que apresentam ainda hipertensão arterial e intolerância à glicose. Evidências recentes não deixam dúvidas de que há um novo e importante mecanismo envolvido no controle da ação dos glicocorticóides, mediada principalmente pela regulação pré-receptor exercida pela 11-beta-hidroxiesteróide-desidrogenase (11ßHSD) $(7,15)$.

Foi demonstrado que animais transgênicos deficientes da 11- $\beta$-HSD1 apresentam perfil metabólico favo- 
rável, com aumento do catabolismo lipídico, redução do nível intracelular de glicocorticóides, aumento da sensibilidade à insulina, HDL-colesterol e apo-AI, sugerindo um fenótipo protetor em relação ao processo aterosclerótico (15). Por outro lado, a administração de glicocorticóides ou a elevação de glicocorticóides endógeno promovem dislipidemia e resistência à insulina. Estes achados clínicos sugerem que a ação dos glicocorticóides tem papel importante na patofisiologia da síndrome metabólica.

\section{GLICOCORTICÓIDE, METABOLISMO E AÇÃO}

O cortisol ou hidrocortisona como é conhecido, é o principal glicocorticóide endógeno. Este hormônio esteróide é produzido e secretado pela zona fasciculada das adrenais e está sob controle do hormônio adrenocorticotrófico (ACTH). Seu nível circulante é regulado pela atividade do eixo hipotálamo-pituitária-adrenal, um circuito de feedback neuroendócrino que pode ser ativado por estímulos fisiológicos, tais como o estresse (16). A ação local dos glicocorticóides é dependente de seu metabolismo intracelular pela 11ßHSD. 11ßHSD1 ativa os glicocorticóides (cortisona para cortisol), enquanto 11ßHSD2 inativa o hormônio. Estas duas isoenzimas são produtos de diferentes genes e têm distinta distribuição nos tecidos. A $11 \beta-H S D 1$ é expressada primariamente no fígado, adipócitos, rim e cérebro, enquanto a $11 \beta$ HSD2 é expressada principalmente nos rins e glândulas salivares (17). Assim, a atividade destas enzimas desempenha papel importante nas ações fisiológicas dos GCs.

A cortisona apresenta-se no plasma de forma livre, no entanto, aproximadamente $6 \%$ do cortisol são carreados pela albumina e $90 \%$ se combinam reversivelmente a uma alfa-globulina, sintetizada no fígado denominada transcortina ou CBG (corticosteroid-binding globulin), funcionando como reserva (18). A CBG está presente em diversos tecidos e pode regular a ação do GC de maneira tecido específico. Por exemplo, níveis significativamente baixos de CBG no tecido adiposo de ratos Zucker contribuem para a resistência à insulina (19).

A ação dos glicocorticóides também é mediada pelo receptor de glicocorticóide (GR), um receptor nuclear que regula eventos fisiológicos diretamente, ativando ou inibindo genes alvos envolvidos na infla- mação, gliconeogênese e diferenciação dos adipócitos (20). Na maioria dos casos, o efeito na transcrição é estimulador, levando ao acúmulo de RNAs mensageiros que codificam a síntese protéica. Sabese que efeitos inibitórios também ocorrem em genes que são regulados negativamente. Entretanto, alguns fatos permanecem obscuros na ação deste hormônio. Portanto, a ação dos GC nos tecidos alvos não é dependente somente da concentração de GC circulante e da expressão celular de GR, mas também do metabolismo intracelular tecido-específico de GC pela $11-\beta-H S D s$.

\section{EFEITOS METABÓLICOS DOS GLICOCORTICÓIDES}

Os glicocorticóides são hormônios com ação antagônica à insulina. Exercem ação predominante sobre o metabolismo intermediário, com efeitos principalmente sobre os tecidos hepático, muscular e adiposo. Numa situação de estresse, os glicocorticóides (no homem, principalmente o cortisol, e na maioria dos roedores, a corticosterona) contribuem na mobilização de substratos energéticos, com a finalidade de recuperar os tecidos lesionados e promover a homeostase orgânica, já que ambos são hormônios catabólicos.

Diferente das ações fisiológicas (isto é reguladoras e metabólicas) dos glicocorticóides de secreção endógena, em altas concentrações como evidenciadas na síndrome de Cushing ou pela administração exógena de seus análogos sintéticos, induzem resistência à insulina e inúmeras desordens metabólicas ao organismo. As alterações metabólicas evidenciadas na obesidade em humanos, que têm relação importante com doenças do coração, incluindo hipertensão, resistência à insulina, diabetes tipo 2 e hiperlipidemia, características da síndrome metabólica, também são encontradas na síndrome de Cushing, quando esta é causada pelo aumento dos níveis circulantes de glicocorticóides (21). Observou-se também que a administração exógena de GCs aumenta os níveis séricos de insulina e triglicérides (22).

Os efeitos metabólicos dos GCs são mediados por diversos mecanismos que são fisiologicamente relevantes no desenvolvimento da resistência à insulina hepática e periférica, dislipidemia, obesidade e hiperglicemia. Suas ações em tecidos específicos contribuem para as anormalidades da síndrome metabólica. 
No músculo esquelético, os glicocorticóides exercem efeitos catabólicos via aumento na proteólise (23), diminuição no transporte de aminoácidos para o interior do músculo (24), inibição da síntese de proteínas (25) e indução da miostatina, um conhecido fator regulador negativo da massa muscular (26). Elevada atividade GC no músculo esquelético pode inibir a via de sinalização da insulina por diversos mecanismos, incluindo inibição da translocação de GLUT4 para a membrana celular (27) e inibição da atividade da Lipoproteína Lipase (LPL) e, conseqüentemente, captação reduzida de triglicérides da circulação (28). Estas ações dos GCs na sinalização e regulação metabólica estão relacionadas com a quantidade de receptores de glicocorticóides nos tecidos sensíveis a insulina e com a disponibilidade de cortisol na forma ativa, que é convertido da cortisona pela enzima 11- $\beta$-HSD1 nas células dos músculos esqueléticos (20). Estudos anteriores detectaram aumento no RNAm dos GR em biópsia de músculo esquelético de diabéticos do tipo 2 , com uma subseqüente diminuição na expressão de GR, correlacionada com melhora na sensitividade à insulina nestes pacientes após tratamento intensivo (29). Estes resultados sugerem que a atividade anormal dos GR no músculo esquelético pode ter um significativo efeito na resistência à insulina observada no diabetes do tipo 2.

Os GCs exercem alterações importantes também no metabolismo hepático. Estes esteróides conduzem à elevação da glicemia, atuando na captação, consumo periférico e produção de glicose (30). Estimulam a gliconeogênese hepática a partir da liberação de ácidos graxos e glicerol dos adipócitos e de aminoácidos provenientes da inibição na síntese protéica periférica $(31,32)$. Especificamente, os GCs induzem a gliconeogênese hepática pela ativação dos receptores de glicocorticóides (GR) dessa via, que estimula a expressão da fosfoenolpiruvato carboxilase (PEPCK) e glicose-6-fosfatase (G6Pase), enzimas chaves da cascata de gliconeogênese (33-34). Isto resulta em aumento da produção hepática de glicose e hiperglicemia.

Estes esteróides interferem no metabolismo das gorduras, favorecendo a lipólise com aumento de ácidos graxos no plasma, decorrente de estímulo de liberação do tecido adiposo, possivelmente por ação per- missiva com outros hormônios, como as catecolaminas e o glucagon (35). Além disso, ativam a lipase hormônio sensível, uma enzima chave na lipólise, inibida pela insulina. No entanto, a secreção endógena ou administração de doses excessivas de glicocorticóides promovem aumento dos depósitos de gordura, principalmente na região abdominal (36). Evidências acumuladas demonstram que a atividade enzimática da $11-\beta-H S D 1$ tem papel importante na patogênese da obesidade visceral e da síndrome metabólica (37). Em modelos de obesidade em roedores (38) a 11- $\beta$-HSD1 encontra-se diminuída no fígado e aumentada no tecido adiposo mesentérico. Além do mais, a reduzida sensitividade à insulina em tecidos periféricos está associada aos efeitos antagonistas do GC na translocação dos transportadores de glicose dos compartimentos intracelulares da membrana plasmática (39). A compreensão dos mecanismos de ação dos glicocorticóides sobre o metabolismo envolve uma série de eventos moleculares com a participação da insulina. Variações nos níveis e/ou graus de fosforilação do IR, IRS-1 e IRS2 e na atividade da PI 3-quinase foram observados em animais submetidos à administração exógena de glicocorticóides $(40,41)$. Similar mecanismo é responsável pela resistência à insulina no músculo esquelético (42), redução na captação de aminoácidos pelos adipócitos estimulada pela insulina (43), além do que o aumento da lipólise ou oxidação de gordura pode estar relacionado à resistência à insulina periférica induzida por GC (44).

O uso de glicocorticóides pode também alterar a funcionalidade da célula beta do pâncreas. Estudos demonstraram que os GCs têm efeito na secreção de insulina da célula beta em animais e alteram o pico deste hormônio no período pós-prandial em humanos (45-46).

Outros efeitos adicionais do excesso de glicocorticóides estão implicados na hipertensão, outra característica comum da síndrome metabólica. Os GCs têm ação agonista com receptores mineralocorticóides (MR), e a sua ativação provoca retenção de sal e elevação da pressão sangüínea. A expressão de ambas, $11-\beta-H S D 1$ e $11-\beta-H S D 2$, no rim sugere que a interconversão dos GCs inativo e ativo aconteça de maneira equilibrada. Assim, a ativação de MR pode ser controlada especificamente em cada tecido (47). 
Contudo, o excesso de GC resultante do aumento da

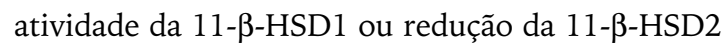
conduz à ativação do MR e hipertensão.

Além disso, a exposição aos glicocorticóides pode provocar alterações nos níveis circulantes de agentes vasodilatadores, como por exemplo, inibição da síntese de óxido nítrico (NO) (48-49). Como o NO liberado do endotélio exerce importante ação vasodilatadora sobre as artérias, a menor síntese desse vasodilatador, em resposta ao efeito inibitório da dexametasona na ativação da enzima óxido nítrico síntase (NOS), provocaria injúria sobre o endotélio e, conseqüentemente, alteração de fluxo sanguíneo, prejuízo na responsividade dos tecidos periféricos à insulina e alteração no metabolismo lipídico, con- tribuindo para o aumento da pressão arterial (50). Este conjunto de dados fisiológicos sugere que os efeitos metabólicos dos glicocorticóides ocorrem em diferentes tecidos e o aumento da ação deles contribui para a etiologia da síndrome metabólica (51) (Figura 1). Satisfatoriamente por meio de estudos moleculares e genéticos, mais informações têm sido disponibilizadas para esclarecer, minuciosamente, a ação dos GCs sob tecidos específicos e sua relação com as características da síndrome metabólica. Diante dessa eminente relação entre glicocorticóides e anormalidades metabólicas, veremos a seguir a maneira pela qual o exercício físico pode auxiliar na homeostase do organismo frente a esta situação.

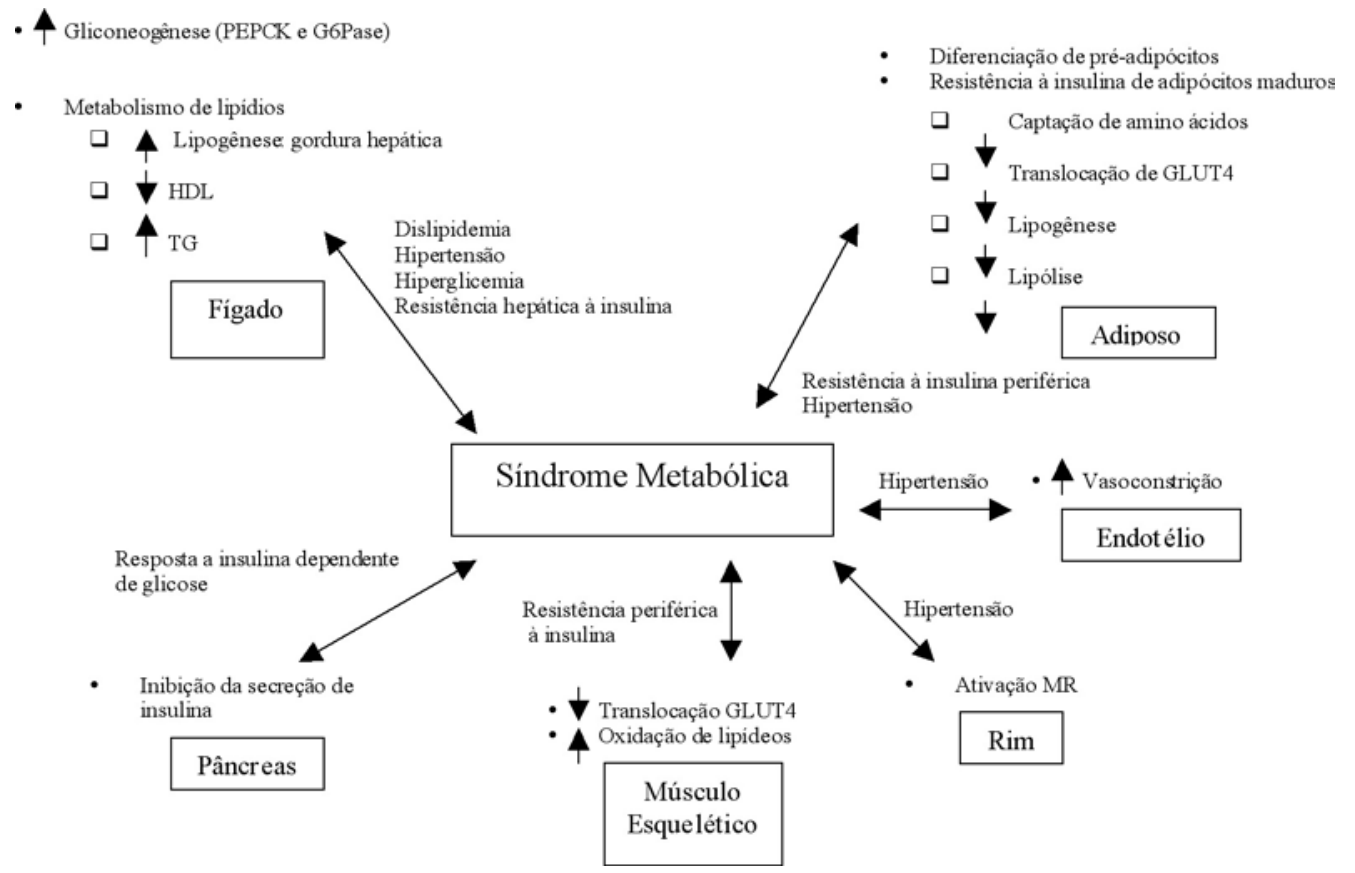

Figura 1. Relação entre os efeitos dos glicocorticóides e as características da síndrome metabólica. Os maiores efeitos nos diferentes tecidos são sumarizados $e$ a associação fisiológica com a síndrome metabólica é apresentada. Adaptado de Wang Minghan [51].

\section{EXERCÍCIO FÍSICO E BENEFÍCIOS À SAÚDE}

O exercício físico tem sido apontado por muitos autores como promotor de bem estar e saúde aos seus praticantes, promovendo melhora na aptidão funcional e contribuindo favoravelmente, com o sistema circulatório, respiratório, imunológico, entre outros, reduzindo os fatores deletérios relacionados ao sedentarismo (52,
$53,54)$. Admite-se atualmente que um estilo de vida fisicamente ativo é um fator de grande importância na redução de uma série de distúrbios, dentre os quais incluem-se os problemas cardíacos, alguns tipos de câncer, diabetes mellitus, obesidade, osteoporose entre outros. Os possíveis benefícios da realização de uma atividade física bem orientada, realizada de maneira 
sistematizada, são muitos e estudos recentes enfatizam a importância de programas de exercícios em longo prazo no tratamento e prevenção das anormalidades metabólicas comuns da síndrome metabólica e de suas complicações $(55,56)$.

\section{MÚSCULO ESQUELÉTICO E METABOLISMO}

O músculo esquelético representa a maior massa de tecido periférico, sumarizando aproximadamente $40 \%$ da massa corporal total, e exerce papel primordial sobre o balanço energético. É responsável por mais de $30 \%$ do dispêndio de energia, e é o tecido primário na captação, disponibilidade e estoque de glicose estimulada por insulina. Além disso, o músculo exerce seu efeito no metabolismo via modulação de lipídios circulantes e de seus estoques. O catabolismo lipídico suprime mais de $70 \%$ do requerimento de energia para o músculo em repouso. No entanto, a necessidade de maior consumo de energia faz com que o exercício físico estimule a captação de glicose, melhore a sensitividade à insulina após o esforço e promova a oxidação de ácidos graxos da circulação, provendo dessa maneira ATP para a musculatura esquelética durante e após a atividade contrátil dos músculos (57).

Estas adaptações, além de serem fundamentais para o rendimento de um atleta, também são importantes em pessoas com anormalidades metabólicas, como evidenciado na obesidade, diabetes do tipo 2 e hipertensão e, em longo prazo, podem diminuir a concentração de glicose sanguínea e melhorar o perfil lipídico, tendo assim, efeito preventivo e retardando o desenvolvimento destas doenças.

Nesse contexto, o músculo esquelético é um importante alvo terapêutico no controle da doença cardiovascular, uma vez que os efeitos favoráveis sobre o metabolismo proporcionados pelo exercício físico aeróbio, que diretamente influenciam os fatores de risco de doenças cardiovasculares, são primariamente dirigidos pelo músculo esquelético. Assim, esta revisão foca os efeitos do exercício físico regular sob os distúrbios metabólicos comumente presentes na síndrome metabólica, como a intolerância à glicose, resistência à insulina, obesidade central, dislipidemia e hipertensão, todos bem documentados fatores de risco para doenças coronarianas, observadas em situações de uso de glicocorticóides.

\section{EXERCÍCIO FÍSICO E RESISTÊNCIA À INSULINA}

O transporte de glicose para os miócitos é agudamente regulado pela insulina e por fatores semelhantes à insulina através da ativação de uma série de proteínas intracelulares (58). Além disso, o transporte de glicose no músculo esquelético é também estimulado por mecanismos independentes de insulina, que são ativados pela contração muscular (59), hipóxia (60), óxido nítrico (61) e bradicinina (62). Apesar de ainda obscuros os caminhos pelos quais estes fatores estimulam a translocação de GLUT-4, recentes evidências suportam o papel da ativação da "5-adenosine monophosphate-activated protein kinase" (AMP kinase), uma enzima ativada pelo decréscimo de energia celular (63) e que discutiremos com mais detalhes à frente. Vejamos a seguir os mecanismos pelos quais o exercício físico promove uma melhora na sensitividade à insulina.

A atividade física regular promove uma melhora na resposta dos eventos pós-receptores da insulina, isto é, na fosforilação de proteínas que iniciam as ações do hormônio (64). Associado a isto ocorre o aumento da síntese de transportadores de glicose e da atividade de enzimas ligadas ao seu metabolismo. Todos estes fenômenos proporcionam melhorias à tolerância à glicose (64). Além dos efeitos moleculares do exercício, o aumento do fluxo sanguíneo pode acarretar maior disponibilidade de insulina para os tecidos periféricos, contribuindo para a melhora metabólica observada durante o treinamento físico.

Os exercícios físicos, principalmente os resistidos que proporcionam a manutenção ou o aumento de massa magra, podem ser importantes na homeostase da glicose, uma vez que proporcionam um aumento do espaço destinado ao estoque desta hexose (glicogênio). Já o aumento do número de fibras de contração lenta (também conhecidas como fibras do tipo 1 ou fibras vermelhas), em resposta ao treinamento aeróbio, favorece a oxidação de glicose.

Além disso, os níveis aumentados de fatores semelhantes à insulina (IGFs) induzida pelos exercícios também podem atuar no transporte de glicose para os músculos em atividade (65), contribuindo para o aumento da captação de glicose.

Portanto, está bem estabelecido que o exercício físico (agudo e crônico) melhora a captação de glicose estimulada pela insulina, tanto em humanos quanto 
em modelos animais $(32,64,65,66)$. O Programa de Prevenção ao Diabetes dos Estados Unidos concluiu que a introdução de um programa com modificações no estilo de vida, incluindo uma redução de $7 \%$ no peso corporal e a realização de, no mínimo, 150 minutos de atividade física semanais, pode, em um período de 3 anos, reduzir a incidência de diabetes tipo 2 em $58 \%$ em indivíduos com significante risco para desenvolver a doença (67).

Além do mais, dados do nosso laboratório demonstraram que ratos administrados com dexametasona por 10 semanas apresentam resistência à insulina. No entanto, os animais que receberam a droga e realizaram exercício físico no mesmo período apresentaram taxa de remoção de glicose durante o teste de tolerância à insulina semelhante ao grupo de animais controles $(4,32)$. Portanto, o exercício regular pode favorecer organismos que apresentam intolerância à glicose e resistência à insulina (como observado no diabetes do tipo 2 , obesidade, excesso de glicocorticóides, entre outras), reduzindo o número de mortes por doenças cardiovasculares.

Apesar desses benefícios comprovados, a realização do exercício por um período curto de tempo está associada a baixa sensibilidade à insulina, enquanto a permanência por períodos longos de inatividade física está associada a um aumento da resistência à insulina $(68,69)$. O efeito do exercício físico sobre a sensibilidade à insulina tem sido demonstrado entre 12 e 48 horas após a sessão de exercício, porém esse efeito parece retornar aos níveis pré-atividade em três e cinco dias após o último período de realização de exercício físico (70), o que reforça a necessidade de adotar-se um estilo de vida fisicamente ativo.

Além disso, muitas vezes é necessário associar dietas e uso de medicamentos para que se possa controlar as anormalidades metabólicas.

\section{EXERCÍCIO FÍSICO, OBESIDADE E DISLIPIDEMIA}

O exercício físico regular produz efeitos no grau de aterosclerose coronária e nos fatores de risco de doenças cardíacas. É observada em diversos estudos uma associação entre exercício e redução da adiposidade, principalmente aquela localizada na região do tronco e do abdomen $(71,72)$. Estudos com animais de experimentação têm demonstrado que o treinamento físico promove redução da gordura corporal.
Nosso grupo verificou recentemente que ratos administrados com dexametasona apresentavam um aumento no peso do tecido adiposo epididimal. Entretanto, os ratos que realizaram exercícios regulares tiveram um menor acúmulo de gordura nessa região quando comparados com o grupo controle e com o grupo que recebeu dexametasona e permaneceu sedentário (32). Este fato talvez possa justificar as observações feitas por outros pesquisadores, que afirmam que o treinamento aeróbio colabora com a perda de gordura $(54,73)$. Tal tentativa de analogia e de interpretação pode ser reforçada pelos resultados obtidos no estudo de Mensink e colaboradores (74), quando ficou evidenciado que a intervenção de um programa de atividades físicas promove melhoras no perfil metabólico de indivíduos diabéticos tipo 2 , além de aumento da capacidade de utilização de ácidos graxos livres pelo músculo esquelético.

Sabe-se que, além do efeito direto no gasto calórico, o exercício físico regular mantem o metabolismo basal aumentado por longo período após as sessões de treinamento. Entre os efeitos importantes da prática de atividades físicas sobre a perda de peso destacam-se o aumento da atividade da enzima lípase hormônio sensível e o aumento da densidade mitocôndrial, potencializando a oxidação de lípidos e favorecendo, assim, o emagrecimento (75). Em relação ao perfil lipídico, há uma grande variabilidade nos resultados encontrados nas pesquisas relacionando níveis lipídicos e exercício, em função da heterogeneidade das populações, do treinamento realizado, do controle do uso de fármacos, da dieta e da perda de peso corporal associada (76). Apesar disso, são muitas as evidências que comprovam os efeitos benéficos do exercício na melhora do perfil lipídico, com o aumento do HDL-colesterol e redução do LDL-colesterol e dos triglicérides $(55,56$, $72,73,74,75,76)$. Portanto, o treinamento físico pode ser uma excelente intervenção não-farmacológica para o tratamento da obesidade e dos seus distúrbios metabólicos.

\section{EXERCÍCIO FÍSICO E HIPERTENSÃO}

Há evidências associando anormalidades da ação da insulina à hipertensão nos pacientes. Por outro lado, o exercício tem sido mostrado como um fator importante na prevenção da hipertensão e de suas doenças 
associadas. Bouchard e colaboradores (77) revelaram uma redução significativa de $3 / 3 \mathrm{mmHg}$ (sistólica/diastólica) em normotensos e uma maior redução em indivíduos hipertensos (em média 10/8 $\mathrm{mmHg}$ ) com a prática regular de exercícios físicos. Semelhantes resultados foram observados por Arroll e Beaglehole (78) que encontraram reduções de 6$7 \mathrm{mmHg}$ na pressão sistólica e diastólica em indivíduos normotensos e hipertensos com o treinamento físico. As alterações da pressão arterial estão associadas com a síntese de óxido nítrico (NO). O NO produzido no endotélio provoca relaxamento das fibras musculares lisas adjacentes, inibe a agregação de plaquetas e a adesão de células inflamatórias à superfície vascular, diminui a proliferação muscular e a apoptose endotelial, aumenta a atividade das enzimas que neutralizam os radicais livres, reduzindo assim, a atividade inflamatória. Em condição de exercício ocorre aumento do débito cardíaco e redistribuição do fluxo sanguíneo para a musculatura esquelética e circulação cardíaca. Este mecanismo é mediado pela enzima óxido nítrico síntase endotelial (eNOS), cuja expressão genética pode ser potencializada com exercícios físicos aeróbios regulares $(79,80)$. Portanto, estímulos como a pressão de arrasto sobre as paredes dos vasos sanguíneos (shear stress) pelo exercício contribui para a geração basal de óxido nítrico no sistema arterial (81). Assim, em conseqüência do exercício, a elevação da produção de óxido nítrico aumenta a vasodilatação dependente do endotélio e inibe os múltiplos processos envolvidos com a aterogênese, bem como o processo inflamatório associado. Dessa forma, o exercício físico pode ser importante para a geração de NO, que é inibida pelo uso de dexametasona (49).

\section{AÇõES MOLECULARES DO EXERCÍCIO FíSICO NO CONTROLE METABÓLICO: NOVAS PERSPECTIVAS} AMPK e transporte de glicose

Apesar de muitos anos de pesquisas, o mecanismo molecular pelo qual o exercício promove estes benefícios permanece não totalmente conhecido. A proteína quinase ativa pelo AMP (AMPK) tem sido recentemente implicada na regulação de muitos destes efeitos. Existe evidência de que esta enzima participa das adaptações crônicas adquiridas pelo treinamento físico no músculo esquelético, através de modificação na expressão de genes de diversas proteínas (82).
A ativação da AMPK durante o exercício é decorrente de um rápido declínio nas razões AMP/ATP e creatina/fosfocreatina do músculo em atividade. Diferentes experimentos têm demonstrado que a contração, efetivamente, aumenta a atividade da AMPK (83). Por exemplo, foi demonstrado que a contração muscular por estimulação elétrica in situ (84) e a contração isolada de músculo de ratos in vitro (85) aumentam significativamente a atividade da AMPK. O exercício de ciclismo também aumenta a atividade da isoforma $\alpha 2$ AMPK em humanos, no entanto, tal ativação é dependente, da intensidade e do tempo de duração do esforço (86). A $\alpha 2$ AMPK é ativada durante o exercício de intensidade moderada quando, em geral, a isoforma $\alpha 1$ AMPK parece ser mais resistente à ativação e é somente ativada em condições extremas, como na contração isolada de músculo de ratos in vitro, durante o sprint no ciclismo e ou nos exercícios supramáximos em humanos (86).

Esse aumento da atividade da AMPK em resposta a uma necessidade de gerar ATP, durante o exercício, promove a translocação das vesículas contendo GLUT-4, facilitando assim o transporte de glicose para o músculo de maneira semelhante à da insulina, embora por cascatas de sinalização diferentes e independentes. Nessa situação, a redução da malonil-CoA permite o aumento da ação da carnitina palmitoiltransferase I (CPT1), que aumenta a eficiência do transporte de ácidos graxos para as mitocôndrias e conseqüente oxidação.

De maneira similar à contração muscular, a ativação da AMPK com 5-amino-4-imidazole-carboxamineribose (AICAR), um ativador farmacológico da AMPK, resulta em um aumento da captação de glicose no músculo, indicando de fato que esta enzima pode ter um papel determinante na entrada de glicose nesse tecido (87). Observou-se que a ativação crônica da AMPK por AICAR diariamente durante 4 semanas, para simular o treinamento com exercícios, foi associada com aumentos no conteúdo de GLUT4 , assim como à atividade das enzimas oxidativas mitocôndriais (88). Esses dados sugerem que repetidas sessões de exercício físico podem gerar adaptações bioquímicas no músculo esquelético, através da ativação repetida da AMPK.

Desse modo, a AMPK tem se tornado um interessante alvo para o desenvolvimento de drogas. Um 
grupo de autores examinaram a ação do exercício em diabéticos tipo 2 (89). A atividade da $\alpha 2$ AMPK no músculo esquelético destes pacientes foi similar aos indivíduos controles do estudo, um indicativo de que indivíduos com essa doença têm um funcionamento normal da AMPK no músculo.

\section{RECEPTORES HORMONAIS NUCLEARES E METABOLISMO}

$A$ atividade de fatores transcripcionais da família de receptores hormonais nucleares (NRs) no músculo esquelético tem ganhado destaque e surge como um potencial alvo no combate contra a obesidade (90), síndrome metabólica (91), diabetes do tipo 2 e dislipidemia (92). A presença de NRs no músculo esquelético, como por exemplo, o receptor ativado por proliferadores de peroxissoma (do inglês "Peroxisome Proliferator-Activated Receptor" - PPAR), com três isoformas conhecidas $\operatorname{PPAR} \alpha, \operatorname{PPAR} \delta$ e $\operatorname{PPAR} \gamma(91,92$, $93,94)$, tem sido mostrado estar envolvido no aumento da disponibilidade de glicose estimulada por insulina, diminuição de triglicerídeos e aumento do catabolismo lipídico, dispêndio energético, efluxo de colesterol e níveis de HDL-c no plasma. Estudo recente, demonstrou que a ativação do PPAR $\delta$ conduz a um fenótipo predominante de fibras de contração lenta (oxidativas) e aumenta, dramaticamente, a capacidade de endurance, aumento da sensitividade à insulina e resistência à obesidade (95).

Conseqüentemente, o entendimento das funções dos NRs no músculo esquelético possui enorme utilidade farmacológica para o tratamento da doença cardiovascular e da síndrome metabólica.

Nesse contexto, o entendimento de como o exercício físico interage com os fatores transcripcionais da família de receptores hormonais nucleares, deve trazer novas descobertas científicas em relação aos efeitos da atividade física sobre as anormalidades metabólicas evidenciadas na síndrome metabólica e no excesso de glicocorticóide.

Portanto, pode-se dizer que o exercício físico regular pode exercer adaptações favoráveis ao organismo e deve ser pensado como forma de tratamento ou prevenção em indivíduos que apresentam resistência à insulina e outras anormalidades metabólicas, evidenciadas tanto na síndrome metabólica quanto pelo uso de glicocorticóides. Assim, o treinamento físico pode auxiliar no tratamento desses distúrbios meta- bólicos, mas não necessariamente normalizar as alterações no metabolismo, devendo, portanto, ser associado a outras condutas quando necessárias.

\section{CORRESPONDÊNCIA}

José Rodrigo Pauli

Rua XV de Novembro, 1701, centro

13400-370 Piracicaba

São Paulo

Brasil

rodrigosere@yahoo.com.br 


\section{BIBLIOGRAFIA}

1. Rosen J, Miner JN. (2005). The search for safer glucocorticoid receptor ligands. Endocrine Reviews 26 (3):452-464.

2. Leclerc N, Luppen CA, Ho VV, Nagpal S, Hacia JG, Smith E, Frenkel B. (2004). Gene expression profiling of glucocorticoid -inhibited osteoblasts. Journal of molecular Endocrinology 33: 175-193

3. Hochberg Z, Pacak K, Chrousos GP. (2003). Endocrine withdrawal syndromes. Endocrine Reviews 24 (4): 523-538.

4. Pauli JR, Almeida Leme JAC, Crespilho DM, Mello MAR, Rogatto GP, Luciano E. (2005). Influência do treinamento físico sobre parâmetros do eixo hipotálamo-pituitária-adrenal de ratos administrados com dexametasona. Rev Port Cien Desp 2: 143-152.

5. Arnaldi G, Angeli A, Atkinson AB, Bertagna X, Cavagnini F, Chrousos GP, Fava GA, Findling JW, Gaillard RC, Grossman AB, Kola B, Lacroix A, Mancini T, Mantero F, Newell-Price J, Nieman LK, Sonino N, Vance ML, Giustina A, Boscaro M. (2003). Diagnosis and complications of Cushing's syndrome: a consensus statement. J Clin Endocrinol Metab 88 (12): 5593-5602.

6. Covar RA, Leung DY, McCormick D, Steelman J, Zeitler P, Spahn JD. (2000). Risk factors associated with glucocorticoid-induced adverse effects in children with severe asthma. J Allergy Clin Immunol (106 (4): 651-659.

7. Watts LM, Manchem VP, Leedom TA, Rivard AL, McKay RA, Bao D, Neroladakis T, Monia BP, Bodenmiller DM, Cao JX-C, Zhang HY, Cox AL, Jacobs SJ, Michael MD, Sloop KW, Bhanot S. (2005). Reduction of hepatic and adipose tissue glucocorticoid receptor expression with antisense oligonucleotides improves hyperglycemia and hyperlipidemia in diabetic rodents without causing systemic glucocorticoid antagonism. Diabetes 54: 1846-1853.

8. Cameron AJ, Shaw JE, Zimmet PZ. (2004). The metabolic syndrome: prevalence in worldwide populations. Endocrinol Metab Clin North Am 33: 351-375.

9. Zimmet P, Alberti KG, Shaw J. (2001). Global and societal implications of the diabetes epidemic. Nature 414:782-87.

10. Eckel RH, Grundy SM, Zimmet PZ. (2005). The metabolic Syndrome. Lancet 365: 1415-1428.

11. Andrew R, Gale CR, Walker BR, Seckl JR, Martyn CN. (2002). Glucocorticoid metabolism and the metabolic syndrome: associations in an elderly cohort. Exp Clin Endocrinol Diabetes 110 (6): 284-290.

12. Rosmond R, Dallman MF, Bjorntorp P. (1998). Stress-related cortisol secretion in men: relationships with abdominal obesity and endocrine metabolic and hemodynamic abnormalities. J Clin Endocrinol Metab 83 (6): 1853-1859.

13. Walker BR, Phillips DI, Noon JP, Panarelli M, Andrew R, Edwards HV, Holton DW, Secld JR, Webb DJ, Watt GC. (1998). Increased glucocorticoid activity in men with cardiovascular risk factors. Hypertension 31 (4): 891-895.

14. Filipovsky J, Ducimetiere P, Eschwege E, Richard JL, Rossein G, Claude JR. (1996). The relationship of blood pressure with glucose, insulin, heart hate, free fatty acids and plasma cortisol levels according to degree of obesity in meddle-aged men. J Hypertens 14 (2): 229-235.

15. Morton NM, Holmes MC, Fievet C. (2001). Improved lipid and lipoprotein profile, hepatic insulin sensitivity, and glucose tolerance in 11 beta-hydroxysteroid dehydrogenase type 1 null mice. J Biol Chem 276: 41293-300.

16. Delbende C, Delarue C, Lefebvre H, Bunel DT, Szafarczyk
A, Mocaer E, Kamoun A, Jegou S, Vaudry H. (1992). Glucocorticoid, transmitters and stress. Br J Psychiatry Suppl 160 (15): 24-35.

17. Walker EA, Stewart PM. (2003). 11 $\beta$-hydroxisteroid dehydrogenase: unexpected connections. Trends Endocrinol Metab 14 (7): 334-339.

18. Weiser JN, Do YS, Feldman D. (1979). Synthesis and secretion of corticosteroid-binding globulin by rat liver. A source of heterogeneity of hepatic corticosteroid-binders. $J$ Clin Invest 63 (3): 461-467.

19. Grasa MM, Cabot C, Balada F, Virgili J, Sanchis D, Monserrat C, Fernandez-Lopez JA, Remesar X, Alemany M. (1998). Corticosterone binding to tissues of adrenolectomized lean and obese Zucker rats. Horm Metab Res 30 (12): 699-704.

20. Whorwood CB, Donovan SJ, Flanagan D, Phillips DL, Byrne CD. (2002). Increased glucocorticoid receptor expression in human skeletal muscle cells may contribute to the pathogenesis of the metabolic syndrome. Diabetes 51: 1066-1075.

21. Reynolds RM, Walker BR. (2003). Human insulin resistance: the role of glucocorticoids. Diabetes Obes Metab 5: 5-12.

22. Freedman MR, Horwitz BA, Stem JS. (1986). Effect of adrenolectomy and glucocorticoid replacement on development of obesity. Am J Physiol 250: R595-R607.

23. Darmaun D, Mathews DE, Bier DM. (1988). Physiological hypercortisolemia increases proteolysis, glutamine, and alanine production. Am J Physiol 255: 366-373.

24. Hasselgren PO, Fisher JE. (1999). Counter-regulatory hormones and mechanisms in amino acid metabolism with special reference to the catabolic response in skeletam muscle. Curr Opin Clin Nutr Metab Care 2: 9-14.

25. Shah, OJ, Anthony JC, Kimball SR, Jefferson LS. (2005). Glucocorticoids oppose translational control by leucine in skeletal muscle. Am J Physiol Endocrinol Metab 279: E1185E1190.

26. Ma K, Mallidis C, Bhasin S, Mahabadi V, Artaza J, Gonzáles-Cadavid N. (2003). Glucocorticoid-induced skeletal muscle atrophy in associated with upregulation of myostatin gene expression. Am J Physiol Endocrinol Metab 285: 363-371.

27. Weinstein SP, Paquin T, Pritsker A, Haber RS. (1995). Glucocorticoid-induced insulin resistance: dexametasone inhibits the activation of glucose transport in rat skeletal muscle by both insulin- and non insulin-related stimuli. Diabetes 44: 441-445.

28. Ong JM, Simsolo RB, Saghizadeh M, Goers JW, Kern PA. (1995). Effects of exercise training and feeding on lipoprotein lipase gene expression in adipose tissue, heart, and skeletal muscle of the rat. Metabolism 44: 1596-1605.

29. Vestgaard H, Bratholm P, Christensen NJ. (2001). Increments in insulin sensitivity during intensive treatment are closely correlated with decrements in glucocorticoid recptor mRNA in skeletal muscle from patients with type II diabetes. Clin Sci 101: 533-540.

30. Schneiter P, Tappy L. (1998). Kinetics of dexamethasoneinduced alterations of glucose metabolism in health humans. Am J Physiol 275: E806-E813.

31. Stojanovska L, Rosella G, Proietto J. (1990). Evolution of dexamethasone-induced insulin resistance in rats. Am J Physiol 258: E748-756.

32. Pauli JR, Gomes RJ, Luciano E. (in press). Hipothalamy- 
pituitary axis: effects of physical training in rats administered with dexamethasone. Neurologia.

33. Friedman JE, Yun JS, Patel YM, Mcgrane MM, Hanson RW. (1993). Glucocorticoid regulate the induction of phosphoenolpyruvate carboxykinase (GTP) gene transcription during dibetes. J Biol. Chem 268 (17): 12952-12957.

34. Argaud D, Zhang Q, Pan W, Maitra S, Pilkis SJ, Lange AJ. (1996). Regulation of rat liver glucose-6-phosphatase gene expression in different nutritional and hormomal states: gene structure and 5'-flanking sequence. Diabetes 45 (11): 1563-1571.

35. Allan EH, Titheradge MA. (1984). Effect of treatment of rats with dexamethasone in vivo on gluconeogenesis and metabolite compartmentation in susequently isolated hepatocytes. Biochem J 219: 117-123.

36. Lima JG, Nóbrega LHC, Nóbrega MLC, Rodrigues Jr AB, Pereira AFF. (2002). Supressão hipotálamo-hipófiseadrenal e risco de insuficiência adrenal secundária ao uso de dexametasona nasal. Arq Brás Endocrinol Metab 46 (2): 193-196.

37. Wake DJ, Rask E, Livingstone DE, Sodeberg S, Olsson T, Walker BR. (2003). Local and systemic impact of transcriptional up-regulation of $11 \beta$-hydroxysteroid dehydrogenase type 1 in adipose tissue in human obesity. J Clin Endocrinol Metab 88 (8): 3983-3988.

38. Kershaw EE, Morton NM, Dhillon H, Ramage L, Seekl JR, Flier JS. (2005). Adipocyte-specific glucocorticoid inactivation protects against diet-induced obesity. Diabetes 54:1023-1031.

39. Oda N, Nakai A, Mokuno T, Sawai Y, Nishida Y, Mano T, Asano K, Itoh Y, Kotake M, Kato S, Masunaga R, Iwase K, Tsujimura T, Itoh M, Kawabe T, Nagasaka A. (1995). Dexamethasone-induced changes in glucose transporter 4 in rat heart muscle, skeletal muscle and adipocytes. Eur J Endocrinol 133 (1): 121-126.

40. Sakoda H, Ogihara T, Anai M, Funaki M, Inukai K, Katagiri H, Fukushima Y, Onishi Y, Ono H, Fujishiro M, Kikuchi M, Oka Y, Asano T. (2000). Dexametasone-induced insulin resistance in 3T3-L1 adipocytes is due to inhibition of glucose transport rather than signal transduction. Diabetes 49 : 1700-1708.

41. Carvalho CRO, Saad MJA. (2002). Resistência à insulina induzida por glicocorticóides: investigação de mecanismos moleculares. Arq Brás Endocrinol Metab 42 (1): 13-21.

42. Dimitriads G, Leighton B, Parry-Billings M, Sasson S, Young M, Krause U, Bevan S, Piva T, Wegener G, Newsholme EA. (1997). Effects of glucocorticoid excess on the sensitivity of glucose transport and metabolism to insulin in rat skeletal muscle. Biochem J 321: 707-712.

43. Grunfeld C, Jones DS. (1986). Glucocorticoid-induced insulin resistance in vitro: inhibition of insulin-stimulated methylaminoisobutyric acid uptake. Horm Metab Res 18 (3): 149-152.

44. Ekstrand A, Saloranta C, Ahonen J, Gronhagen-Riska C, Groop LC. (1992). Reversal of steroid-induced insulin resistance by a nicotinic-acid derivative in man. Metabolism 41 (7): 692-697.

45. Lambillote C, Gilon P, Henquin JC. (1997). Direct glucocortioid inhibition of insulin secretion. An in vitro study of dexamethasone effects in mouse islets. J Clin Invest 99 (3): 414-423.

46. Hollingdal M, Juhl CB, Dall R, Sturis J, Veldhuis JD,
Schmitz O, Porksen N. (2002). Glucocorticoid induced insulin resistance impairs basal but not glucose entrained high-frequency insulin pulsatility in humans. Diabetologia 45 (1): 49-55.

47. Lloyde-Macgilp SA, Nelson SM, Florin M, Lo M, McKinnell J, Sassard J, Kenyon CJ. (1999). 11ß-hydroxysteroid dehydrogenase and corticosteroid action in lyon hipertensive rats. Hypertension 34 (5): 1123-1128.

48. Fletcher AJW, McGarrigle HHG, Edwards CMB, Fowden AL, Giussani DA. (2002). Effects of low dose dexamethasone treatment on basal cardiovascular and endocrine function in fetal sheep during late gestation. J Physiol 542 (2): 649-660.

49. Severino C, Brizzi P, Solinas A, Secchi G, Maioli M, Tonolo G. (2002). Low-dose dexamethasone in the rat: a model to study insulin resistance. Am J Physiol 283: E367-373.

50. Viaro F, Nobre F, Evora PRB. (2000). Expressão das óxido nítrico sintetases na fisiopatologia das doenças cardovasculares. Arq Brás Cardiol 74 (4): 365-379.

51. Wang M. (2005). The role of glucocorticoid action in the pathophysiology of the metabolic syndrome. Nutr Metab 2 (3): 1-14.

52. Pauli JR, Souza LS, Gobbi S, Zago AS. (2005). Efeitos de um programa de treinamento físico personalizado sobre a aptidão funcional, composição corporal e bioquímica sanguínea em idosas. Motricidade 1 (2): 116-125.

53. Pauli JR, Souza LS, Zago AS, Gobbi S. (2004). The effects of a physical activity program in a 12-year period, in older people. J Aging Phys Activ 12 (3): 452-453.

54. Boulé NG, Kenny GP, Haddad E, Wells GA, Sigal RJ. (2003). Metaanalysis of the effect of structured exercise training on cardiorespiratory fitness in type 2 diabetes mellitus. Diabetologia 46: 1071-1081.

55. Guimarães GV, Ciolac EM. (2004). Síndrome metabólica: abordagem do educador físico. Rev Soc Cardiol Estado de São Paulo 14 (4): 130-142.

56. Ciolac EM, Guimarães GV. (2004). Exercício físico e síndrome metabólica. Rev Bras Med Esporte 10 (4): 319-324.

57. Hargreaves M, Cameron-Smith D. (2002). Exercise, diet, and skeletal muscle gene expression. Med Sci Sports Exerc 34 (9): 1505-1508.

58. Zierath JR, Krook A, Walberg-Henriksson H. (2000). Insulin action and insulin resistance in humans skeletal muscle. Diabetologia 43: 821-835.

59. Nesher R, Karl IE, Kipnis DM. (1985). Dissociation of effects of insulin and contraction on glucose transport in rat epitrochlearis muscle. Am J Physiol Cell Physiol 249:C226-C232.

60. Azevedo JL, Carey JO, Pories WJ, Morris PG, Dohm GL. (1995). Hypoxia stimulates glucose transport in insulinresistant human skeletal muscle. Diabetes 44: 695-698.

61. Balon TW, Nadler JL. (1997). Evidence that nitric oxide increases glucose transport in skeletal muscle. J Appl Physiol 82: 359-363.

62. Mann WR, Villauer EB, Barilla D, Battle B, Dunning BE, Balkan B. (1995). Effects of bradykinin on glucose metabolism in isolated rat soleus muscle and on blood glucose levels in ob/ob mice. Diabetes 44: 133A.

63. Kurth-Kraczek EJ, Hirshman MF, Goodyear LJ, Winder WW. (1999). 5' AMP-activated protein kinase activation causes GLUT4 translocation in skeletam muscle. Diabetes 48: 1667-1671. 
64. Luciano E, Carneiro EM, Carvalho CRO, Carvalheira JBC, Perez SB, Reis MAB, Saad MJA, Boschero AC, Velloso LA. (2002). Endurance training improves responsivenes to insulin and modulates insulin signal transduction through the phosphatidylinositol 3-Kinase/ Akt-1 pathway. Eur J Endocrinol 12 (2): 202-209.

65. Gomes RJ, Caetano FC, Mello MAR, Luciano E. (2005). Effect of Chronic Exercise on Growth Factors in Diabetic Rats. Journal of Exercise Physiology 8 (2): 16-23.

66. Pauli JR, Rodrigues Júnior JC, Antunes DFR, Luciano E. (2003). Treinamento físico e administração de insulina: efeitos sobre o metabolismo de carboidratos e proteínas. Motriz 9 (2): 71-74.

67. Diabetes Prevention Program Research Group. (2002). Reduction of the incidence of type 2 diabetes with lifestyle intervention or metformin. N Engl J Med 346: 393-403.

68. Kump DS, Booth FW. (2005). Alterations in insulin receptor signalling in the rat epitrochlearis muscle upon cessation of voluntary exercise. J Physiol 562 (3): 829-838.

69. Booth FW, Chakravarthy MV, Spangenburg EE. (2002). Exercise and gene expression: physiological regulation of the human genome through activity. J Physiol 543 (2): 399-411.

70. Mikines KJ, Sonne B, Farrel PA. (1988). Effect of physical exercise on sensitivity and responsiveness to insulin in humans. Am J Physiol 254: E248-259.

71. Stubbs CO, Lee JA. (2004). The obesity epidemic: both energy intake and physical activity contribute. MJA 181 (9): 489-491.

72. Hardman AE. (1996). Exercise in the prevention of atherosclerotic, metabolic and hypertensive diseases: a review. J Sports Sci 14: 201-218.

73. Francischi RP, Pereira LO, Lancha Júnior AH. (2001). Exercício, comportamento alimentar e obesidade: revisão dos efeitos sobre a composição corporal e parâmetros metabólicos. Rev Paul Educ Fís 15 (2):117-140.

74. Mensink M, Blaak EE, Vidal H, Brun TWA, Glatz JFC, Saris WHM. (2003). Lifestyle changes and lipid metabolism gene expression and protein content in skeletal muscle of subjects with impaired glucose tolerance. Diabetologia 46: 1082-1089.

75. Seip RL, Semenkovich CF. (1998). Skeletal muscle lipoprotein lipase; molecular regulation and physiological effects in relation to exercise. Exerc Sport Sci Rev 26: 191-218.

76. French AS, Story M, Jeffery RW. (2001). Environmental influences on eating and physical activity. Annu Rev Public Health 22: 309-335.

77. Bouchard C, Shephard RJ, Stephens T. (eds). (1994). Physical activity, fitness and health: Internacional proceeedings and consensus statement. Champaign: Human Kinetics.

78. Arroll B, Beaglehole R. (1992). Does physical activity lower blood pressure? A critical review of the clinical trials. Journal of Clinical Epidemiology 45: 439-447.

79. Shen W, Zhang X, Wolin MS, Sessa W, Hintze TH. (1995). Nitric oxide production and NO synthase gene expression contribute to vascular regulation during exercise. Med Sci Sports Exerc 8: 1125-1134.

80. Kingwell BA. (2000). Nitric-oxide-mediated regulation during exercise: effects of training in health and cardivascular disease. FASEB J 14: 1685-1696.

81. Tanabe T, Maeda S, Miyauchi T, Iemitsu T, Takanashi M, Irukayama-Tomobe Y, Yokota T, Ohmori H, Matsuda M.
(2002). Exercise training improves ageing-induced decreased in eNOS expression of the aorta. Acta Physiol Scand 178 (1): 3-10.

82. Wojtaszewski JFP, Birk JB, Frosig C, Holten M, Pilegaard H, Dela F. (2005). 5'AMP activated protein kinase expression in human skeletam muscle: effects of strenght training and type 2 diabetes. J. Physiol 564 (2): 563-573.

83. Mcgee SL, Howlett KF, Starkie RL Cameron-mith D, Kemp BE, Hargraves M. (2003). Exercise increases nuclear AMPK $\alpha 2$ in numan skeletal muscle. Diabetes 52: 926-928.

84. Hutber CA, Hardie DG, Winder WW. (1997). Electrical stimulation inactivates muscle acetyl-CoA carboxylase and increases AMP' activated protein kinase. Am. J. Physiol 272 (2): E262-266.

85. Hayashi T; Hirshman MF; Fujii N; Habinowski SA; Witters LA; Goodyear LJ. (2000). Metabolic stress and altered glucose transport activation of AMP-activated protein kinase as a unifying coupling mechanism. Diabetes 49 (4): 527-531.

86. Stephens TJ; Chen ZP; Canny BJ; Michell BJ; Kemp BE; McConell GK. (2002). Progressive increases in human skeletal muscle AMPK alpha 2 activity and ACC phosphorylation during exercise. Am J Physiol Endocrinol Metab 282 (3): E688-E694.

87. Musi N, Yu H, Goodyear LJ. (2002). AMP-activated protein kinase regulation and action in skeletal muscle during exercise. Biochemical Society Transactions 31: 191-195.

88. Winder WW, Holmes BF, Rubink DS, Jensen EB, Chen M, Holloszy JO. (2000). Activation of AMP-activated protein kinase increases mitochondrial enzymes in sckeletal muscle. J Appl Physiol 88: 2219-2226.

89. Musi N, Fujii N, Hirshiman MF. (2001). AMP-activated protein kinase (AMPK) is activated in muscle of subjects with type 2 diabetes during exercise. Diabetes 50: 921-927.

90. Lau P, Bailey P, Dowhan DH, Muscai GE. (1999).

Exogenous expression of a dominant negative RORRalpha 1 vector in muscle cells impairs differentiation: RORalpha 1 directly interacts with p300 and myoD. Nucleic Acids Res 27: 411-420.

91. Tanaka T, Yamamoto J, Iwasaki S, Asaba H, Hamura H, Ikeda Y. (2003). Activation of peroxisome proliferator-activated receptor delta induces fatty acid beta-oxidation in skeletal muscle and attenuates metabolic syndrome. Proc Natl Acd Sci 100: 15924-15929.

92. Henever AL, He W, Barak Y, Le J, Bandyopadhyay G, Olson P. (2003). Muscle-specific Pparg deletion causes insulin resistance. Nat Med 9: 1491-1497.

93. Muscat GE, Wagner BL, Hou J, Tangirala RK, Bischoff E, Rohde P. (2002). Regulation of cholesterol homeostasis and lipid metabolism in skeletal muscle by liver X receptor. J Biol Chem 277: 40722-40728.

94. Muio DM, Way JM, Tanner CJ, Winegar DA, Kliewer SA, Houmard JA. (2002). Peroxisome proliferator-activatred receptor-alpha regulates fatty acid utilization in primary human skeletal muscle cells. Diabetes 51: 901-909.

95. Wang YX, Zhang CL, Yu RT, Cho HK, Nelson MC, BayugaOcampo CR. (2004). Regulation of muscle fiber type and running endurance by PPARdelta. Plos Biol 2:e294. 